\title{
Mean-field resonance-theoretic view of benzenoid networks
}

\author{
D. J. Klein* and T. Ivanciuc \\ Texas A\&M University @ Galveston, Galveston, TX 77553, USA \\ E-mail: kleind@tamug.edu
}

(received 8 Aug 05; accepted 3 Nov 05; published on the web 8 Nov 05)

\begin{abstract}
A simple classically based "mean field" resonance-theoretic approach is described to anticipate the effects of various modes of electron pairing (as in $\pi$-bond formation) for general "alternant" benzenoid conjugated $\pi$-networks. This simplified approach avoids generation and manipulation of individual resonance structures, whence application is facilitated, even for very large systems - so large that there might be hundreds or millions or moles or even substantially greater numbers of resonance structures. Some of the predictions so facilitated for several general circumstances are conveniently manifested as explicit algebraic formulas for numbers of unpaired electrons. The simplicity (and apparent reliability) of these algebraic formulas is emphasized; they involve no more than counts of different "kinds" of $\pi$-center carbons, e.g., the numbers of $\pi$-centers of different functionalities (primary, secondary, or tertiary) which are "starred" (in an "alternant” system) as well as those which are "unstarred". The argumentation also provides some information about rough locations for the (unpaired) spin densities, and ultimately also about the presence of low-lying excited states, magnetic moments, reactivities, and more. In particular these easily used ideas, which are extensions of those already familiar in "classical” organic chemistry should then aid in nano-technological developments.
\end{abstract}

Keywords: Resonance theory, electron pairing, unpaired electrons, spin density, bond localization, conjugated networks, benzenoid molecules, benzenoid polymers, benzenoid nanostructures, defected graphite

\section{Introduction}

Professor Alexandru Balaban (or just Sandy to his friends) has contributed greatly to experimental organic chemistry. But also he has contributed significantly to different theoretical organic programs, and in particular, he and his numerous colleagues have made significant contributions to:

- the development and application of various “topological indices” for QSAR and QSPR methods ${ }^{1,2,3}$; 
- the generation and characterization of networks for degenerate modes of molecular rearrangement ${ }^{4,5,6}$;

- the enumeration of (classical) structures in several molecular classes, e.g., of alkane isomers ${ }^{7,8}$ or of annulene valence isomers ${ }^{9}$;

- $\quad$ the identification of novel organic-chemical structures, e.g., of elemental carbon ${ }^{10,11,12,13}$;

- the utilization of Kekule (neighbor-paired) structures in application ${ }^{14,15,16}$ to conjugated $\pi$ networks, particularly the benzenoids.

And there is more in each of these areas as well as others.

Hence it seems of value to indicate some further work overlapping one of the categories here chosen as the category related to Kekule structures, though somewhat related to the elemental carbon category also. It is here emphasized in application to (neutral) benzenoids, how classical chemical bonding ideas as moderated through Pauling's resonating valence-bond (VB) theory ${ }^{17,18,19,20}$ are useful even at a qualitative level to make novel predictions for radicals, for polymers, and for defected (or decorated) graphites. That is, such classically related ideas are emphasized to be of relevance beyond the realm of the non-radicaloid small molecules for which they were first developed. Such qualitative explication without extensive mathematics or computer usage seems of value in that it is perhaps not presently well recognized by the experimental organic-chemical community just how useful these "classical" ideas are in dealing with new areas of interest in nanotechnology, and how this consequent insight might be used in the design of novel materials.

\section{Conceptual framework}

The present development is formulated with reference to benzenoid-like $\pi$-networks and is phrased pictorially, in consonance with traditional resonance theory. We view there to be a single $\pi$-electron of spin up or spin down on each $\pi$-center, and the resonance structures are viewed as pairing patterns between these spins. Such electron-pairing patterns are long known in the case of benzene.

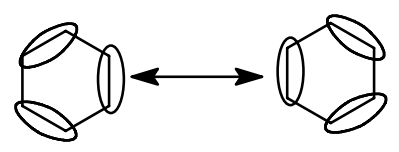

But also we deal with resonance structures for radical cases, such as $m$-xylylene:

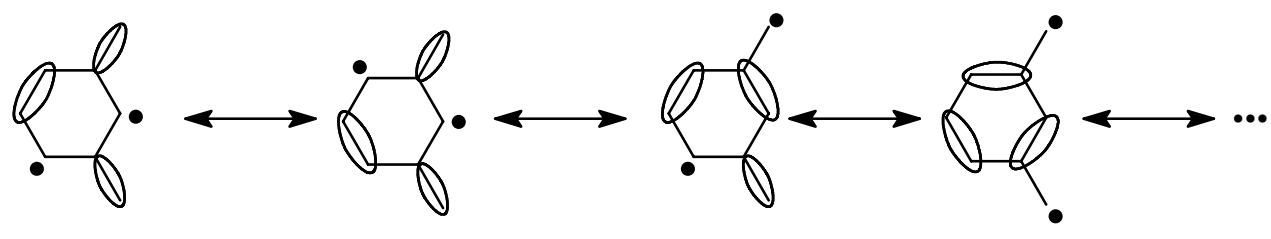


which have unpaired spins. Indeed we address both radicaloid and non-radicaloid resonance structures especially in polymers and large extended systems, as are often of interest in materials science and nanotechnology. Here if allowance of a very few but non-minimal number of unpaired electrons gives very many additional resonance structures, then these (often called “excited”) structures might significantly contribute to the (ground-state) description - and if the unpaired electrons are very delocalized they can turn out to be not so reactive, in the sense that the "free valence" on any given site can then be very small.

It is of convenience especially for larger systems to introduce an "average" way to think about resonance structures, and thereby avoid a detailed generation and consideration of all the resonance structures. This is of use in that there can be overwhelmingly large numbers or resonance structures, even with limitation to the fully paired Kekule structures. E.g., for buckminsterfullerene $\mathrm{C}_{60}$ there are ${ }^{21} 12500$ Kekule structures, and for the icosahedral-symmertry $\mathrm{C}_{180}$ fullerene there are $^{22}$ over a trillion (i.e., 1,389,029,765,625) Kekule structures. For a buckytube with a 6-ring cyclic polyacene unit repeated $L$ times along the length there are ${ }^{23}$ (asymptotically) $\approx A \cdot 6^{L}$ Kekule structures, with $A$ an $L$-independent constant depending on the terminal cap structure, so that for a relatively short buckytube of length $L=100$ one finds something on the order of $10^{78}$ Kekule structures. Thence we wish to avoid the traditional generation of explicit resonance patterns, as in the two illustrations of the preceding paragraph.

The considered simplified formulation ${ }^{24,25,26}$ is most readily made to alternant systems in which the conjugated centers may be divided into starred and unstarred subsets such that neighbors of a site in one of these sets are always in the other set. Then in consonance with classical chemical-bonding ideas, stability is to be enhanced in accordance with three stability criteria:

(0) alternancy pairing - Lower-energy states entail pairings solely between sites of different types (starred and unstarred).

(1) pairing locality - Stability is enhanced by more pairings of neighbor spins, and the stabilization rapidly falls toward 0 as the distance between spins increases.

(2) resonance - Stability is enhanced by greater extents of spin-pairing delocalization (or resonance) amongst different pairs of atoms.

The alternancy criterion (0) is suggested from quantum mechanical VB-theoretic considerations $^{27,28,29,30}$ but really was complicit in early classical ideas such as those of Thiele ${ }^{31}$, and even to some extent in those of Claus ${ }^{32}$, Armstrong ${ }^{33}$, and Bamberger ${ }^{34}$. It is not always viewed as a "stability" criterion in that in addition to the low-energy states, many high-energy states are also encompassed. A quantification of the pairing-locality criterion (1) simply counts the number of nearest neighbor pairings (and at a finer level of detail one could attend to the nearness of a structure with non-neighbor pairing to another structure modified to be more fully neighbor paired). A quantification of the resonance criterion (2) is more delicate, but it is noted that particularly the last two criteria can act either in concert or in opposition, depending on the structural circumstances. That is, there generally is a balance to be considered. Pairing may be 
allowed between non-neighbor sites, though there is a cost to this in sacrificing neighbor pairings [as in a Kekule structur e) which contributes to energy stabilization (i.e., to resonance energy)]. To understand better the balance between local pairing and resonance we introduce some average or "mean-field" quantities. We let $p_{i j}$ be a resonance-theoretic bond order between sites $i \& j$ (of opposite types) as a fraction of resonance structures in the considered ground state having a pairing between sites $i \& j$. Then the sum

$$
v_{i}=1-\sum_{j} p_{i j}
$$

may be identified as a resonance-theoretic free valence, it being understood that the $p_{i j}$ are nonnegative with a sum at each site $\leq 1$, so that each free valence $v_{i}$ satisfies $0 \leq v_{i} \leq 1$, and is viewable as the portion of the $\pi$-valence which is unsatisfied at site $i$. The stability-enhancing tendency (1) for local pairing is manifested as larger values of $p_{i j}$ for nearer lying spins, and the resonance tendency (2) is manifested as comparable $p_{i j}$ for different $j$ adjacent to $i$, i.e, $p_{i j} \approx 1 / d$ if site $i$ is of degree $d$ (with $d=3$ in graphite and bucky-tubes, while $d=2$ in polyenes, aside from the ends where $d=1$ ). Pauling ${ }^{35}$ proposed that with $i \& j$ restricted to neighbors, that $p_{i j}$ be the fraction of maximally paired resonance structures with a $\pi$-bond between sites $i \& j$. Such a 0 order $p_{i j}$ is known ${ }^{35,36,37,38}$ to rather closely correlate with experimental bond lengths, of closed shell (non-radicaloid) species. Sometimes we relax this (0-order) neighbor restriction, and consider the $p_{i j}$ for non-neighbor $i \& j$ (perhaps without recourse to a detailed consideration of the resonance structures). A major point of interest emphasized ${ }^{24,25,26}$ previously is that for defected graphite the demand that the $p_{i j} .1 / 3$ for nearest neighbors in the bulk of the material can imply that the $v_{i}$ are (effectively) non-zero in the region of the defect. This result is strengthened on noting that if $v_{i}$ is restricted so that only sites $j$ within a certain distance are summed upon, then this is really an effective free-valence in that any pairing between distant sites generally should contribute little to stability - i.e., the unpairing of spins paired only at a great distance in an exact ground-state should cost little energy. In particular, neglecting pairing over bulk distances is surely reasonable in considering a meaningful free valence.

\section{Application to small molecules}

The ideas outlined in the preceding section seem in close consonance with standard presentations in organic text-books even of 40 years ago, but subtly differ, perhaps most notably in the emphasis on (Pauling) bond orders $p_{i j}$ and free valences $v_{i}$, which we in fact address without explicit reference to individual resonance structures. The application to small-molecule systems is perhaps less interesting, since then it is often feasible to set down the various resonance structures, and since some ambiguity remains without recourse to a quantitative computational formulation. Still this might be a nice place to start to see that the results agree with the resonance-structure explicit descriptions. From the preceding section we use the stability criteria (0), (1), \& (2) along with the $p_{i j} \& v_{i}$. 
For the classic case of benzene, we seek to assign bond orders and free valences independently of reference to explicit resonance structures. This is easily done:
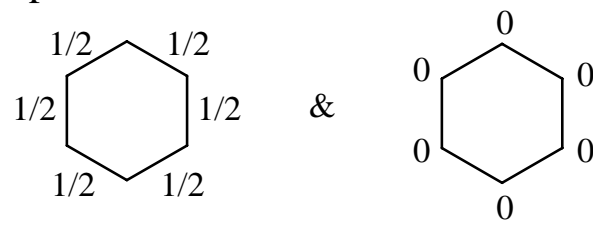

with the first diagram giving bond orders assigned in a symmetric manner such that they sum to 1 at each site (to satisfy criterion (1)), whence free valences of the second diagram are all 0 . And this agrees completely with Pauling bond orders (based on explicit consideration of the two Kekule structures), and hence agrees with the consequent distribution of bond lengths and also trivial spin densities of 0 on every site.

For the case of trimethylene-methane the assignment of bond orders and free valences is also straightforward:

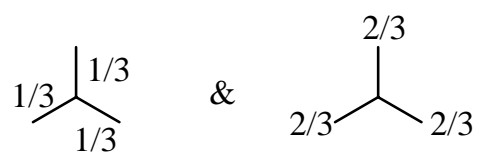

This also agrees completely with Pauling bond orders and the distribution of unpaired spins. The $2 / 3$ of an unpaired electron on each terminal site cannot be paired to one another under the alternancy-pairing condition (0) of pairing solely between sites of the same type (starred or unstarred), so that there are 2 fully unpaired spins and the ground state is a spin triplet. The result here also agrees largely with numerous $a b$ initio quantum-chemical computations $^{39,40,41,42,43,44,45}$, as well as experiment ${ }^{46,47,48}$.

For the case of naphthalene, the assignment is less straightforward, primarily with a degree of ambiguity of assignment of explicit values of bond-orders giving full pairing. Thence we illustrate three (of a range) of the possible choices:
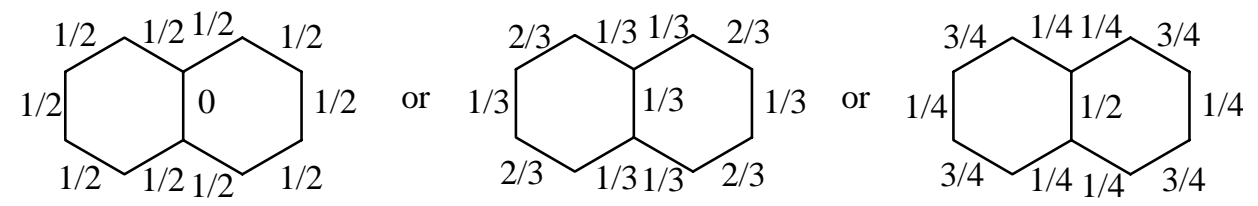

Here it is easy to achieve full pairing (i.e., all $v_{i}=0$ ) by having the bond orders incident at any vertex add to 1 , and this is in agreement with other approaches, but it is only in weak qualitative agreement as to the manifestation of some degree of bond localization.

Bond-order ambiguity as for naphthalene actually occurs fairly frequently in small molecules so long as the stability criteria (0), (1), (2) are used only in a qualitative fashion as here. And so far there seems to be no saving of effort compared to the explicit listing of resonance structures. 
Notably, this often, and perhaps even typically, turns out to be somewhat different for extended structures (as discussed in the next section).

But, even in the small-molecule regime, some notable general results follow with the current simplified approach. In particular from the criteria (0) \& (1) one sees that pairing occurs between starred and unstarred sites to a maximum extent, though the pairing may end up between sites at a great distance. That is, the overall net number of electrons unpaired (at any distance) in an alternant molecule $B$ is

$$
u(B)=\left|\#_{*}(B)-\#_{0}(B)\right|
$$

where $\#_{*}(B) \& \#_{0}(B)$ are the respective numbers of starred and unstarred sites in $B$. That is, the ground-state spin is predicted to be $S=\left|\#_{*}(B)-\#_{0}(B)\right| / 2$. Moreover, this is in precise agreement with the surmise of Ovchinnikov ${ }^{9}$ as well as with rigorous theorems both for ${ }^{50,51,52}$ the full covalent-space Pauling-Wheland VB model and for ${ }^{53}$ the Hubbard model, which is a slightly simplified Parisier/Parr/Pople model - and the theorems consider exact solutions rather than SCF approximations. One might further pursue some general predictions as to where the ground-state unpaired spin-density tends to be localized (on the type of sites in excess) or as to the presence of low-lying excited states (if the paring is weak, being between very distant sites). We now proceed to larger systems.

\section{Extended systems}

For extended conjugated networks the three stability criteria often provide surprisingly strong results. A further general long-held idea is often also used, namely: saturation principle -properties saturate, in that they manifest dependencies only on local (or nearby) substructures. Indeed this is often implicit in much chemical reasoning, e.g., including the idea of functional groups in organic (and even inorganic) chemistry. That is, if the behavior of functional groups depended strongly on distant structural features, then they would not be so extremely important. We now proceed through several examples of the application of the simple mean-field resonance-theoretic argumentation.

As a first case consider poly-para-phenylene, which has been of much interest, with there being much experimental work on this polymer ${ }^{54,55,56,57,58}$. Several different manners of termination of a chain may be imagined, two of which are:

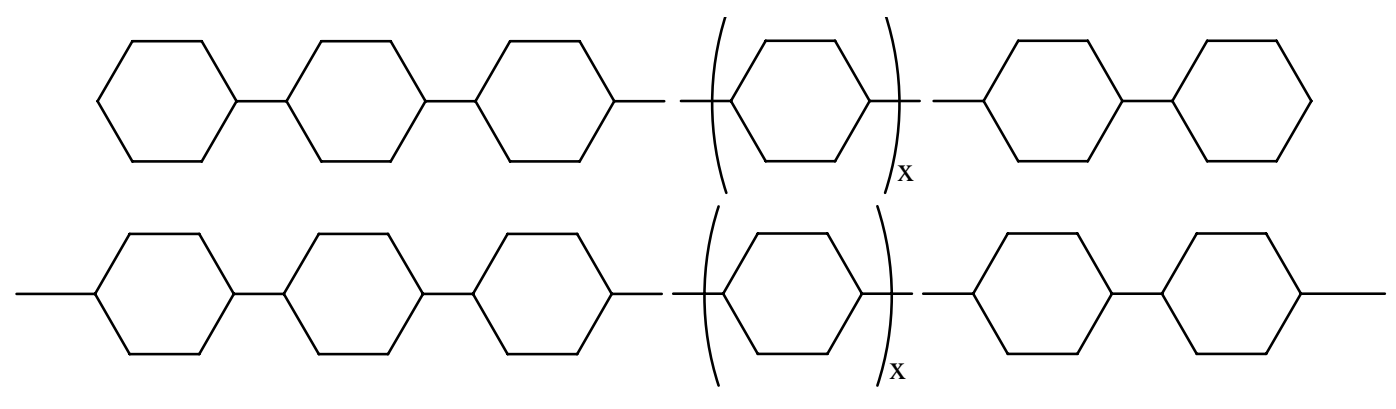


In the first case with so-called "undefected" ends, one reasonably imagines each ring along the chain should be involved in a resonating (or aromatic) sextet, much as in benzene - and in Clar's nomenclature ${ }^{59}$ such a system is termed "fully benzenoid". Thence one imagines bond orders of $1 / 2$ around each ring, with free valences of 0 on each site. But the second "defected" case above is more interesting. If one were to insist on adherence to local (nearest neighbor pairing with all free valences 0 ), then one would end up with a structure

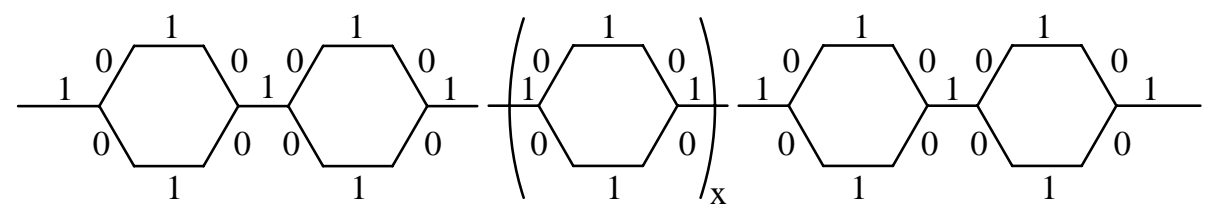

in which no adjacent pair of bonds both have non-zero bond orders so that there evidently is no resonance. But this has incorrectly assumed the complete dominance of the pairing locality criterion (1) over the resonance criterion (2). In fact at least for sufficiently long poly-paraphenylene chains the resonance criterion may be expected to dominate at the cost of a single neighbor pairing, as for the structure.

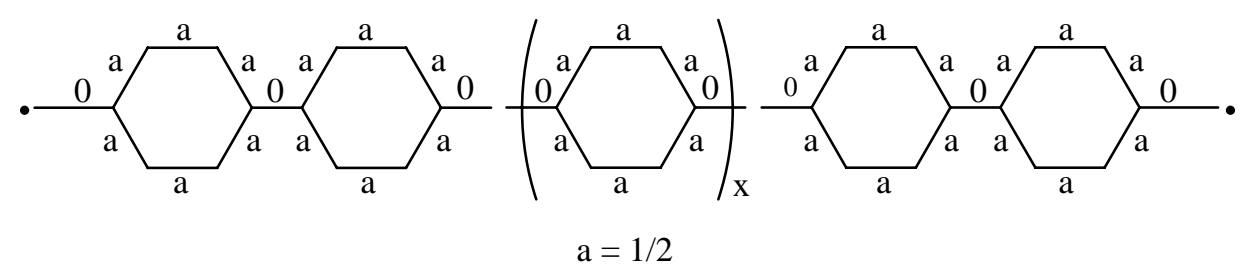

That is, one now has resonance in every single one of the many rings, and only two locally unpaired electrons on the opposite ends of the chain. These two end electrons though locally unpaired, in fact pair to one another to give a singlet ground state - but this pairing being so distant is very weak (in accord with the pairing-locality stability criterion (1)). That is, there should be a very low-lying (triplet) state with these two electrons truly unpaired. And all this is correct, as judged from a variety of theoretical treatments ${ }^{25}$. Here one may view the "saturation principle" to imply that the phenylenes well away from the ends do not know whether the end is "undefected" or not, so that they take the lowest energy local pairing pattern, like that of benzene. Finally it should be commented that locations for all the unpaired electron spin density in the defected structure need not all be entirely on the terminal atoms but could be delocalized a bit especially into the terminal rings as for example with the bond-order assignments

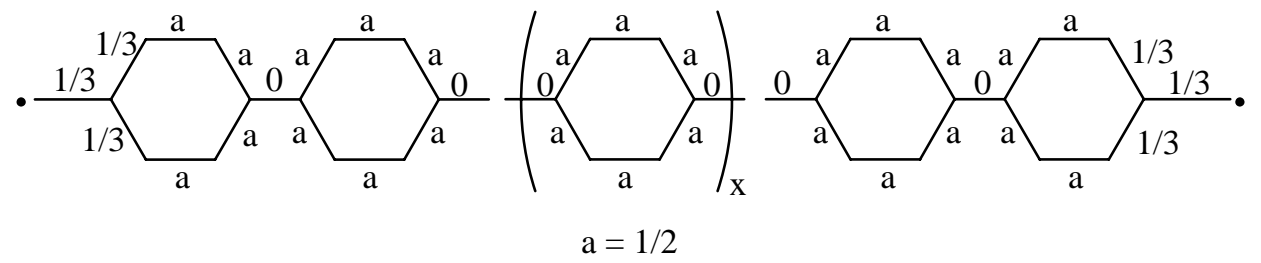


leaving 2/3 of an unpaired electron on the two terminal sites, and 1/6 of an electron on each of the next-nearest neighbors to these end sites. Here it is emphasized that so long as the rings of the bulk near the middle of the chain adopt a pattern as in benzene, the net unpaired spin density if not at each end then near each end has a net value of 1 , as illustrated in the last two illustrations. That is, making the center portion of the chain look like benzene precludes any pairing pattern satisfying the stability criteria other than those with one essentially unpaired electron near each end.

A second example is that of semi-infinite graphite with a single long "globally straight" boundary, for which there are many possibilities, including the so-called "zig-zag” boundary of alternating degree-2 and degree-3 atoms. Full pairing is possible, though then the interior does not appear as in bulk graphite with bonds in each of the 3 directions equivalent. So if one attends to the competition between resonance (criterion (2)) and local pairing (criterion (1)), it is seen that the bulk interior must manifest full resonance, just as for bulk ("edgeless") graphite, with bond-orders of $1 / 3$ in each direction from an atom up to or near the boundary. As a consequence one finds a plausible pattern for free valence being

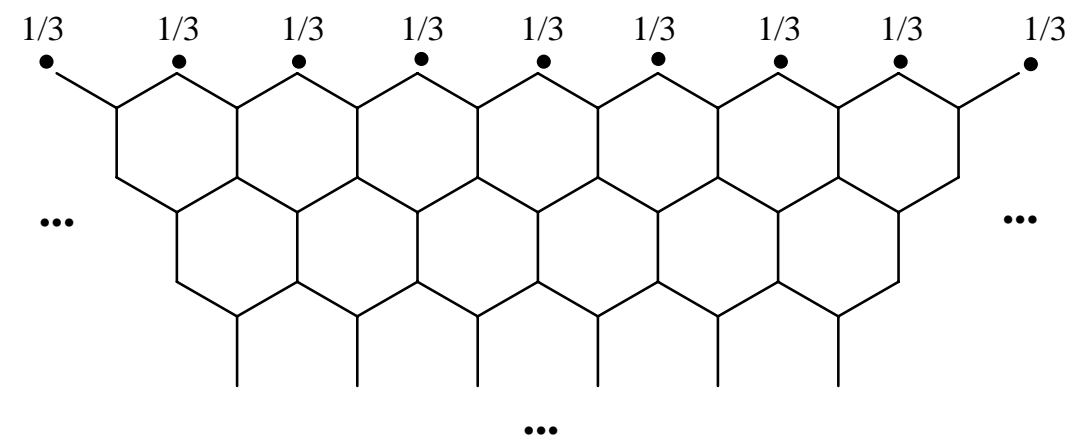

with a net of $1 / 3$ of an unpaired electron shown on each aceneic point of the zig-zag boundary and all being sites of the same type, the "alternancy pairing" criterion (0) tells us that they do not pair to one another. Again this is an extreme approximation as to spin-density location, with it reasonably being that there is some delocalization of the unpaired electrons (or spin density) a little into the region near the boundary (but remaining largely on the same type of sites) with the net unpaired spin density being $1 / 3$ of an electron per unit cell of edge. Also again this prediction seems to be perfectly correct from different theoretical schemes ${ }^{24,60,61}$, with there also being supporting experimental evidence from tunnelling electron microscopy ${ }^{62}$.

Another example of a graphitic boundary is that which looks like a corrugated "arm-chair" boundary, and has a repeating pattern of two degree-2 sites followed by two degree- 3 vertices. Here one still imagines that the bulk of the interior is like bulk boundary-less graphite, though now this does not imply any unpaired spin density near the boundary as seen from the pairing pattern 


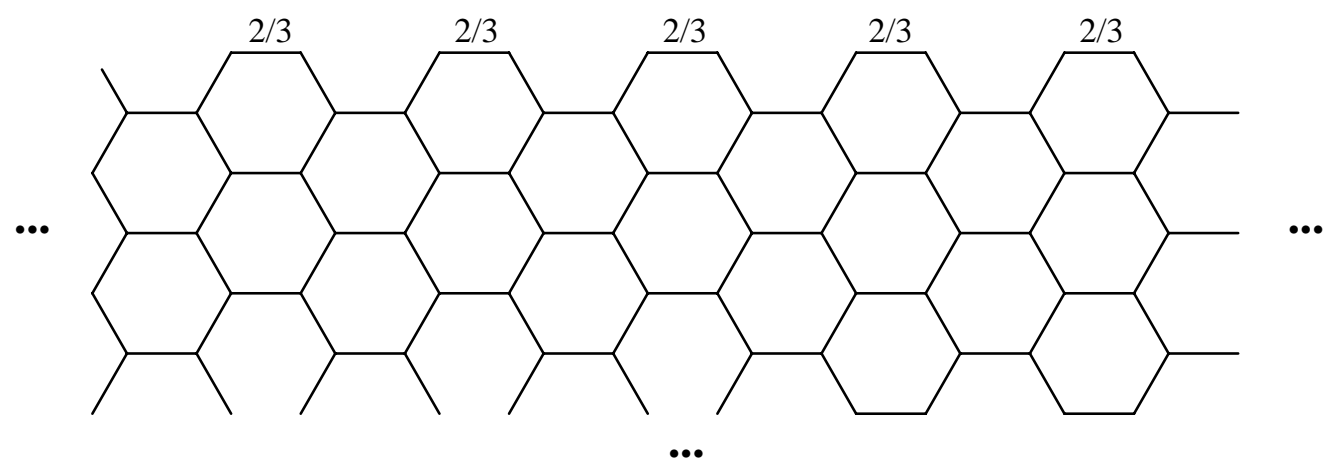

where the bulk of the bonds with values of $1 / 3$ have not been explicitly so labelled, so as to keep the figure "cleaner". Here the bond orders near the boundary are not necessarily exactly correct but might take modified non-1/3 values with associated partial "bond localization" extending a short distance away from the boundary, but approaching the uniform-1/3 values of bulk graphite in the interior. Again there remains no unpaired electrons, and the result agrees with different theoretical treatments ${ }^{24,63,64,65}$ as well as experiment ${ }^{62}$.

Such results for different sorts of translationally symmetric graphitic boundaries are really fairly easily established within the simple qualitative resonance theory outlined, and in fact through it one may make ${ }^{24,25,26}$ a general prediction. If $\#_{n^{*}} \& \#_{n o}$ denote the numbers of degree- $n$ starred and unstarred vertices per repeat unit along the boundary, then the number of unpaired electrons per unit cell is

$$
u_{\text {cell }}=\left|\left(2 \#_{1 *}+\#_{2 *}\right)-\left(2 \#_{10}+\#_{20}\right)\right| / 3
$$

Moreover this has been checked ${ }^{24,25,26}$ against self-consistent UHF-Hubbard model computations $^{24,25,26,64,66,67,68,69}$ for about two dozen different boundaries, with full agreement.

A rather general result is also possible for vacancy defects in graphite. That is one or more contiguous sites are imagined missing from an extended graphitic structure leaving a hole of some shape. For instance, with a single-atom hole one predicts the bulk of the bond orders are $1 / 3$ so that if this is continued all the way in to the defect this leaves $1 / 3$ of an unpaired electron on each of the 3 adjacent sites, 


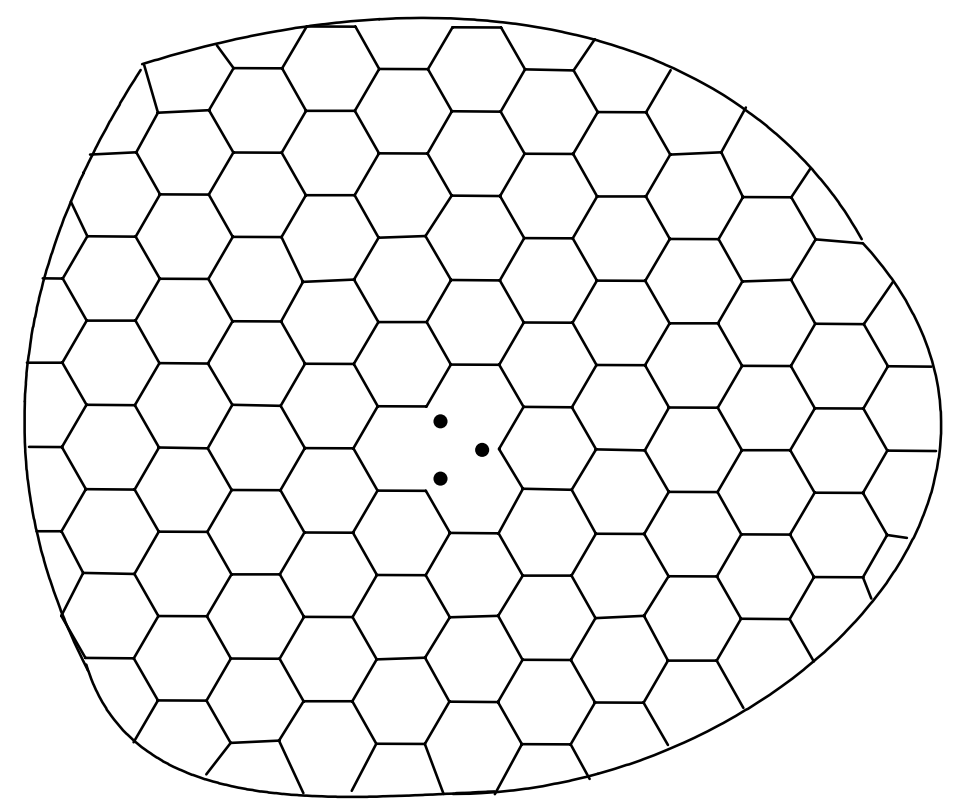

here marked with dots. Again one expects there is some delocalization of the unpaired electron away from the defect, though again there is a net of 1 unpaired electron. And again this is in agreement with various theoretical studies ${ }^{25,26,70,71,72,73,74}$, as well as some experimental electron tunnelling microscopy measurements ${ }^{75,76,77}$. A generally sized and shaped hole may be viewed to arise from deletion of some benzenoid molecular network $R$ so that we might denote the so defected graphite by $G-R$. Then the general prediction ${ }^{25,26}$ for the number $u(G-R)$ of unpaired electrons localized in the area of the defect $G-R$ is

$$
u(G-R)=\left|n_{*}(R)-n_{0}(R)\right|
$$

where the right-hand side is just what was earlier identified as the number of unpaired electrons in a molecule $R$. Really this just says that the local pairing of the deleted molecule being incomplete would when inserted in the graphitic vacancy $G-R$ be such that there would be pairing between $R \& G-R$ such as to precisely accommodate one another and leave perfect graphite. That is, $G-R$ is sort of an anti-molecule to $R$. Further $R$ need not be connected - or equivalently $R$ could represent a pair of molecules, though one then needs to take care in assigning $*$ \& o sites of $R$ to coincide with that for the underlying parent graphite net. Again for about two dozen cases this has been checked ${ }^{25,26}$ against UHF-Hubbard results for defects in large benzenoid sections of 2000 sites (simulating vacancy-defected graphite).

\section{Defected graphitic boundaries}

A further type of example, which does not seem to have been previously considered involves graphitic boundaries which are translationally symmetric along their length except for a single defect. One example involves a locally defected graphitic arm-chair boundary. That is, at one 
location along an infinitely long arm-chair boundary either append or delete a few carbons such that the whole remains a subset of infinite graphite. For example, one could attach a single site as in the structure

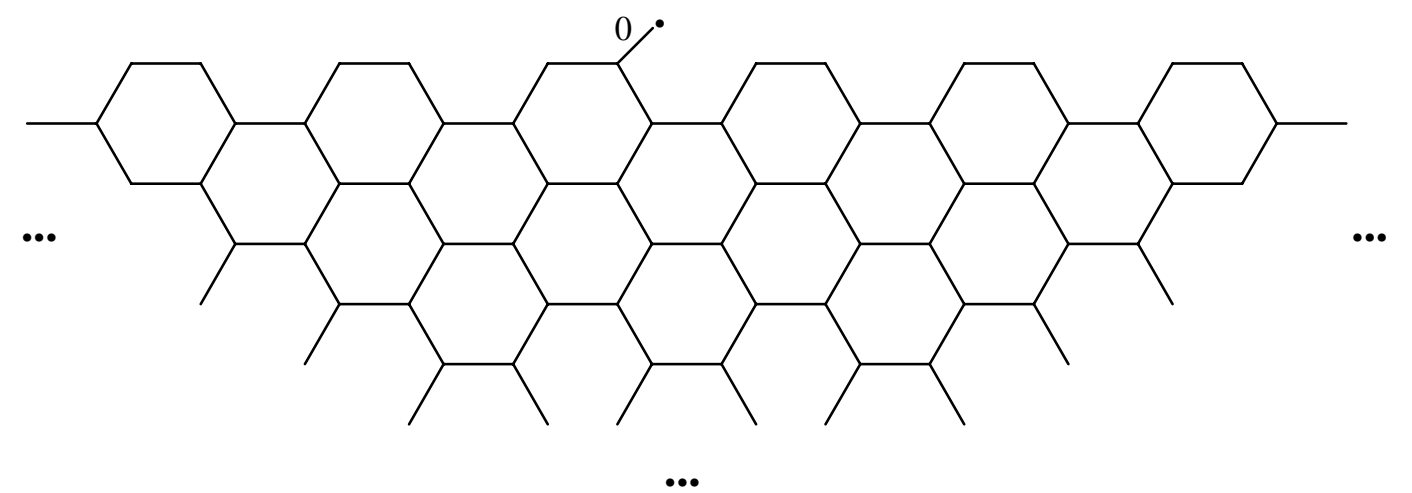

where the bond orders of all the unlabelled bonds are (to our 0th approximation) as for the undefected arm-chair boundary. The added defect bond is given $p_{i j}=0$ since using the perfect arm-chair bond-orders for the other bonds there is no free valence on the base site to pair to the new site. Of course this new bond will not actually have bond order 0 , with however the same net free valence spread out in the local neighborhood. That is, one unpaired electron remains in the region of this boundary defect.

On the other hand one or more sites along the boundary might be missing. For example, consider a single site deleted as

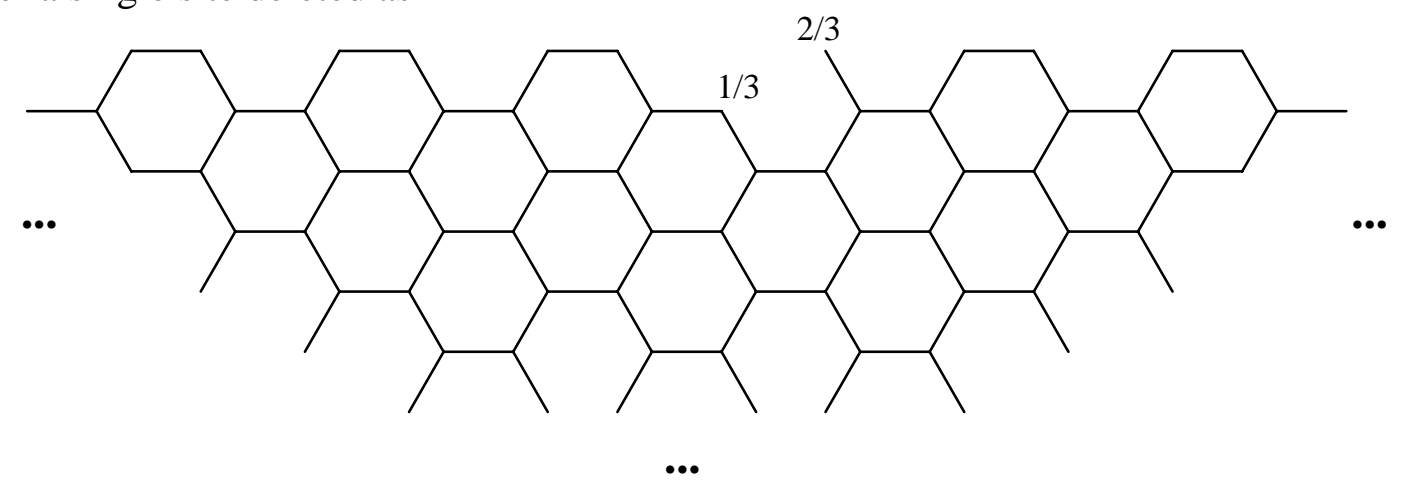

where (in 0-order) we view all bond orders as in the parent undefected arm-chair boundary, whence we identify $1 / 3 \& 2 / 3$ of an unpaired electron on the two indicated (same type) sites of respective degrees $2 \& 1$. That is, one predicts a net of one unpaired electron in the region of this vacancy defect.

An example involving both addition and deletion, of single sites, is 


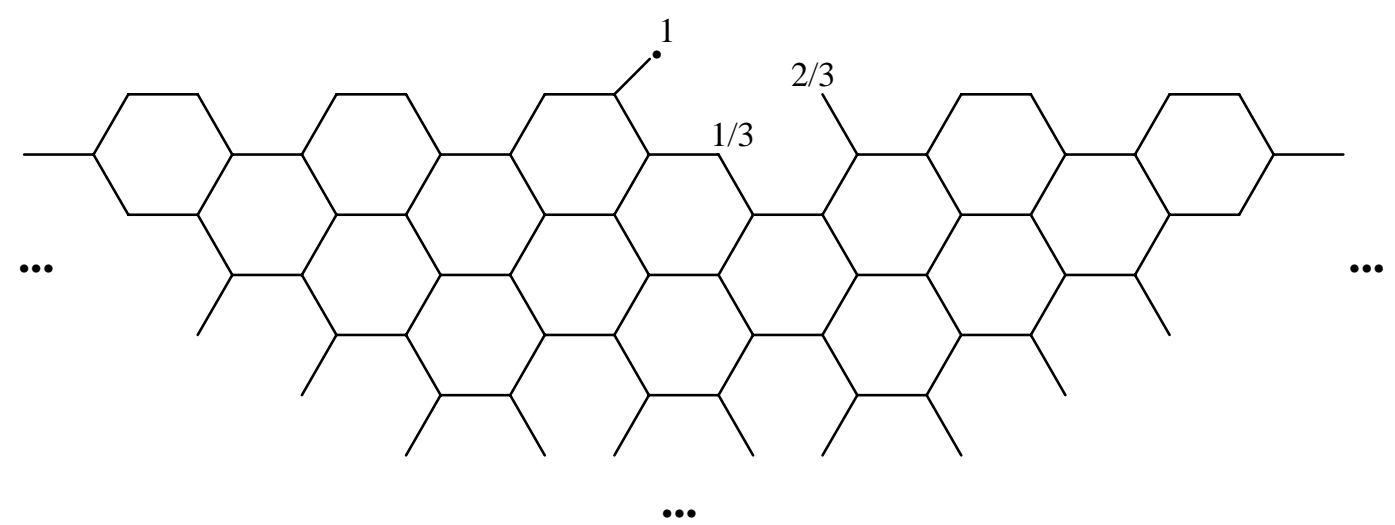

Here the 0 -order free valences are indicated assuming that all bonds of the parent arm-chair edge are just as in this parent. Note that the unpaired spin on the added site is on the type (say *) of site opposite to that (then o) of those sites with $1 / 3 \& 2 / 3$ of an unpaired electron, so that by the pairing-locality criterion (1) there is non-neighbor spin pairing, and the overall net unpaired spin is 0 . Again the bond orders are undoubtedly modified from our 0 -order picture, though when non-neighbor pairing is taken into account there remains a net spin density of 0 . If instead the deleted site were in a different location so that if it were of the type (o) opposite to that of the added site (and the unpaired electron introduced associated to the deleted site would be located on sites of the same type $*$ as the unpaired electron associated to the added site), then there would be a net of two unpaired electrons.

More generally the sets of added and deleted sites may be imagined to correspond to benzenoid molecules $B_{+} \& B_{-}$, with the resulting defected graphitic boundary being denoted $G+B_{+}-B_{-}$. Then via our general mode of argumentation the number of unpaired electrons in the region near the defect is predicted to be

$$
u\left(G+B_{+}-B_{-}\right)=\left|\left[\#_{*}\left(B_{+}\right)-\#_{*}\left(B_{-}\right)\right]-\left[\#_{0}\left(B_{+}\right)-\#_{0}\left(B_{-}\right)\right]\right|
$$

where the choice of $* \&$ o sites in $B_{+} \& B_{-}$is taken to coincide with that for the full graphite network in which the whole is embedded. The argument is really more general than the armchair boundary, so long as the parent translationally symmetric boundary to be defected is such that $u_{\text {cell }}=0$.

For locally defected otherwise translationally symmetric boundaries with $u_{\text {cell }}>0$, the argumentation can also be made. But there is either cancellation or addition of the unpaired electrons depending on how the attachment or deletion is made.

We know of no quantitative computations yet to test any of these predictions, though with the success of all the other similar predictions, the present predictions might be surmised to be correct.

Defects along the way on conjugated polymer chains may also be treated, most simply if the parent polymer is not radicaloid (i.e., has no unpaired electrons in each unit cell in a long chain). But again if there is bulk radicalicity in the polymer chain this also can be treated with not overly complicatedly deducible additive or cancellative effects. 


\section{Prognosis}

Evidently for a diverse range including even extended species qualitative resonating valencebond predictions can be successfully made in a simplified "mean-field" mode. Such classically related ideas developed for (small) molecules seem to apply quite neatly to extended systems. In the preceding 3 sections explicit formulas for numbers of unpaired electrons are given in the 4 displayed equations for 4 different general circumstances:

- molecular alternants (equation (1));

- translationally symmetric boundaries in graphite (equation (2));

- $\quad$ vacancy defects (anti-molecules) in graphite (equation (3)); and

- local defects on translationally symmetric graphitic boundaries (equation (4)).

The formulas are very simple, though evidently quite correct. And the argumentation reveals some information about the location of spin density, with some further consequences which have not been pursued so much here. The great ease and apparent universal success in application to benzenoid structures recommends these ideas to be of much wider consideration, especially in the context of extended nano-structured systems where predictions by other methods seem so often to entail notable computational effort and nuisance. One might entertain ${ }^{78}$ mean-field resonance-theoretic applications beyond benzenoid-based structures, possibly even to selected inorganic structures, in as much as resonance theory has achieved ${ }^{19,79,80,81,82,83,84,85,86,87}$ some success in such areas. It appears to be a delicate matter to extend the present argumentation to general non-alternants (because of the centrality of the alternancy pairing criterion (0)) or heteroatom species (because of the consequent relevance of ionic resonance structures).

The unpaired electrons in the various cases considered of course have different physicochemical manifestations, influencing magnetic susceptibilities and ESR spectra. They also should influence chemical reactivities, though the common predeliction of viewing the presence of unpaired electrons as marks of reactive radicals might need some modification in extended systems in which the unpairedness is greatly spread out. In this regard note that graphite has a zero HOMO-LUMO gap (or band gap), though typically it is not viewed as intrinsically radicaloid. When the unpaired electron density spreads out over an extended area, it can be anticipated to affect electrical conductivities, and even otherwise it should significantly affect dielectric properties. But all this is appropriate for a more quantitative modelling - resonating VB theory being of great interest in such a quantitative context because of the diverse qualitative successes here adumbrated.

The pursuit of further qualitative and quantitative resonating VB theory evidently is a worthwhile goal. It seems that simple theories such as have so engaged Sandy Balaban have a promising future, even in dealing neatly with new chemical areas such as nano-structures.

The authors acknowledge the support (through grant BD-0894) from the Welch Foundation of Houston, Texas. 


\section{References}

1. Balaban, A. T.; Motoc, I. Commun. Math. Chem. (MATCH) 1979, 5, 197.

2. Balaban, A. T. Pure \& Appl. Chem. 1983, 55, 199.

3. Devillers, J.; Balaban, A. T. Topological Indices and Related Descriptors in QSAR and QSPR, Gordon \& Breach: NY, 1999.

4. Balaban, A. T.; Farcasiu, D.; Banica, R. Rev. Roum. Chim. 1966, 11, 1205.

5. Balaban, A. T. Graph theoretical approaches to chemical reactivity; Bonchev, D.; Mekenyan, O. Eds.; Kluwer Academic Pub.: Dordrecht, 1994; pp. 137-180.

6. Balaban, A. T.; Zivkovic, T. P.; Klein, D. J.; Schmalz, T. G. J. Molec. Struct. (TheoChem) 1997, 389, 265.

7. Balaban, A. T. Croat. Chem. Acta 1978, 51, 35.

8. Robinson, R. W.; Harary, F.; Balaban, A. T. Tetrahedron 1976, 32, 355.

9. Balaban, A. T.; Banciu, M.; Ciorba, V. Annulenes, benzo-, hetero-, homo-derivatives and their valence isomers; CRC Press, Boca Raton, FL, 1987.

10. Balaban, A. T.; Rentia, C. C.; Cuipitu, E. Rev. Roum. Chim. 1968, 13, 231.

11. Merz, K. M. Jr.; Hoffmann, R.; Balaban, A. T. J. Am. Chem. Soc. 1987, 109, 6742.

12. Balaban, A. T.; Klein, D. J.; Liu, X. Carbon 1994, 32, 357.

13. Balaban, A. T.; Seitz, W. A.; Klein, D. J. Bull. Soc. Chim. Belg. 1995, 104, 525.

14. Balaban, A. T.; Tomescu, I. Commun. Math. Chem. (MATCH) 1983, 14, 155.

15. Cyvin, S. J.; Balaban, A. T. Struct. Chem. 1991, 2, 485.

16. Balaban, A. T.; Randic, M. J. Chem. Inf. Comp. Sci. 2004, 44, 50.

17. Pauling, L. J. Chem. Phys. 1933, 1, 280.

18. Pauling, L.; Wheland, G. W. J. Chem. Phys. 1933, 1, 362.

19. Pauling, L. The Nature of the Chemical Bond; Cornell U. Press, Ithaca: NY, 1939.

20. Wheland, G. W. Resonance in Organic Chemistry; Wiley: NY, 1955.

21. Klein, D. J.; Schmalz, T. G.; Hite, G. E.; Seitz, W. A. J. Am. Chem. Soc. 1986, 108, 1301.

22. Klein, D. J.; Seitz, W. A.; Schmalz, T. G. Nature 1986, 323, 703.

23. Klein, D. J.; Zhu, H.-Y. Disc. Appl. Math. 1996, 67, 157.

24. Klein, D. J.; Bytautas, L. J. Phys. Chem. 1999, 103, 5196.

25. Ivanciuc, O.; Bytautas, L.; Klein, D. J. J. Chem. Phys. 2002, 116, 4735.

26. Ivanciuc, O.; Klein, D. J.; Bytautas, L. Carbon 2002, 40, 2063.

27. Rumer, G. Nach. Gesselschaft Wissen. Gottingen, Math.-Phys. Klasse 1931, 337.

28. Rumer, G.; Teller, E.; Weyl, H. Nach. Gesselschaft Wissen. Gottingen, Math.-Phys. Klasse 1931, 499.

29. Hartmann, H. Zeit. Naturforschung A 1947, 2, 259.

30. Malrieu, J. P.; Maynau, D. J. Am. Chem. Soc. 1982, 104, 3021.

31. Thiele, J. Ann. Chemie 1898, 306, 125.

32. Claus, A. Theoretische Betrachtungen und deren Anwendung zur Systemik der organischen Chemie; Frieburg, 1866. 
33. Armstrong, H. E. J. Chem. Soc. 1887, 51, 258.

34. Bamberger, E. Ber. 1891, 24, 1758.

35. Pauling, L. The Nature of the Chemical Bond $3^{\text {rd }}$ Edn; Cornell U. Press: Ithaca, NY, 1960; pp 232-238.

36. Ham, N. S.; Ruedenberg, K. J. Chem. Phys. 1958, 29, 1215.

37. Pauling, L. Acta Cryst.B 1980, 36, 1898.

38. Herndon, W. C.; Ellzey, M. L. J. Am. Chem. Soc. 1974, 16, 6631.

39. Yarkony, R.; Schaeffer, H. F. J. Am. Chem. Soc. 1974, 96, 3754.

40. Hashimoto, K.; Fukotome, H. Bull. Chem. Soc. Japan 1981, 54, 3651.

41. Feller, D.; Davidson, E. R.; Borden, W. T. Israel J. Chem. 1982, 23, 105.

42. Lahti, P. M.; Berson, A. R. J. Am. Chem. Soc. 1985, 107, 2273.

43. Prasad, B. L. V.; Radhakrishnan, T. P. J. Phys. Chem. 1992, 96, 9232.

44. Pittner, J.; Nachtigall, P.; Carsky, P.; Hubac, I. J. Phys. Chem. A 2001, 105, 1354.

45. Datta, S. N.; Mukherjee, P.; Jha, P. P. J. Phys. Chem. A 2003, 107, 5049.

46. Platz, M. S.; McBride, J. M.; Little, R. D.; Harrison, J. J.; Shaw, A.; Potter, S. E.; Benson, J. A. J. Am. Chem. Soc. 1976, 98, 5725.

47. Quast, H.; Bieber, L.; Danen, W. C. J. Am. Chem. Soc. 1978, 100, 1306.

48. Platz, M. S.; Carrol, G.; Pierrat, F.; Zayas, J.; Auster, S. Tetrahedron 1982, 38, 777.

49. Ovchinnikov, A. A. Theor. Chim. Acta 1978, 47, 297.

50. Lieb, E. H.; Mattis, D. C. J. Math. Phys. 1962, 3, 749.

51. Klein, D. J. J. Chem. Phys. 1982, 77, 3098.

52. Klein, D. J.; Nelin, C. J.; Alexander, S. A.; Matsen, F. A. J. Chem. Phys. 1982, 77, 3101.

53. Lieb, E. H. Phys. Rev. Lett. 1989, 62, 1201.

54. Baughman, R. H.; Bredas, J. L.; Chance, R. R.; Elsenbaumer, R. L.; Schacklette, L. W. Chem. Rev. 1982, 82, 209.

55. Duke, C. B.; Paton, A.; Slaneck, W. R. Mol. Liq. Cryst. 1982, 83, 177.

56. Grimsdale, A. C.; Mullen, K. Chem. Record 2001, 1, 243.

57. McDiarmid, A. G. Angew. Chem. Intl. Ed. 2001, 40, 2581.

58. Heeger, A. J. Angew. Chem. Intl. Ed. 2001, 40, 2591.

59. Clar, E. The Aromatic Sextet; John Wiley \& Sons: NY, 1971.

60. Seitz, W. A.; Klein, D. J.; Schmalz, T. G.; Garcia-Bach, M. A. Chem. Phys. Lett. 1985, 115, 139.

61. Wakabayashi, K.; Fujita, M.; Kusakabe, K. Czech. J. Phys. 1996, 46, 1865.

62. Giunta, P. L.; Kelty, S. P. J. Chem. Phys. 2001, 114, 1807.

63. Hite, G. E.; Metropoulos, A.; Klein, D. J.; Schmalz, T. G.; Seitz, W. A. Theor. Chim. Acta 1986, 69, 369.

64. Klein, D. J. Reports Molec. Theory 1990, 1, 91.

65. Nakada, K.; Fujita, M.; Wakabayashi, K.; Kusakabe, K. Czech. J. Phys. 1996, 46, 2429.

66. Klein, D. J. Chem. Phys. Lett. 1994, 217, 261.

67. Nakada, K.; Fujita, M.; Dresselhaus, G.; Dresselhaus, M. S. Phys. Rev. B 1996, 54, 17954. 
68. Fujita, M.; Wakabayashi, K.; Nakada, K.; Kusakabe, K. J. Phys. Soc. Japan 1996, 65, 1920.

69. Radovic, L. R.; Bockrath, B. J. Am. Chem. Soc. 2005, 127, 5917.

70. Coulson, C. A.; Herraez, M. A.; Leal, M.; Santos, E.; Senet, S. Proc. Royal Soc. London A 1963, 274, 461.

71. Coulson, C. A.; Poole, M. D. Carbon 1964, 3, 275.

72. Tyutyulkov, N. N.; Madjarova, G.; Dietz, F.; Mullen, K. J. Phys. Chem. B 1998, 102, 10183.

73. Lee, K. H.; Causa, M.; Park, S. S. J. Phys. Chem. B 1998, 102, 6020.

74. Hjort, M.; Stafstrom, S. Phys. Rev. B 2000, 61, 14089.

75. Rong, Z. Y. Phys. Rev. B 1994, 50, 1839.

76. Hahn, J. R.; Kang, J.; Song, S.; Jeon, I. C. Phys. Rev. B 1996, 53, 1725.

77. Atamny, F.; Spillecke, O.; Schlogl, R. Phys.Chem.-Chem.Phys. 1999, 1, 4113.

78. Klein, D. J.; March, N. H. Intl. J. Quantum Chem. 2001, 85, 327.

79. Pauling, L. Proc. Royal Soc. London A 1949, 196, 343.

80. Pauling, L. Proc. Natl. Acad. Sci. USA 1975, 72, 4200.

81. Malrieu, J. P.; Maynau, D.; Daudey, J. P. Phys. Rev. B 1984, 30, 1817.

82. Anderson, P. W. Science 1987, 235, 1196.

83. Kivelson, S. Phys. Rev. B 1987, 36, 7237.

84. Alexander, S. A.; Klein, D. J. J. Am. Chem. Soc. 1988, 110, 3401.

85. Klein, D. J.; Schmalz, T. G.; Garcia-Bach, M. A.; Valenti, R. Phys. Rev. B 1991, 43, 723.

86. Garcia-Bach, M. A. Chem. Phys. Lett. 2001, 345, 183.

87. Calzado, C. J.; Malrieu, J. P. Phys. Rev. B 2001, 63, 214520. 